\title{
Surgery Requiring Detailed Preoperative Simulation and Scar De-epithelialization to Repair Severe Postoperative Scarring from Gastroschisis
}

\author{
Naohiro Ishii ${ }^{1}$, Tomito Oji ${ }^{2}$, Kazuo Kishi ${ }^{2}$ \\ ${ }^{1}$ Department of Plastic and Reconstructive Surgery, Tochigi Cancer Center, Tochigi; ${ }^{2}$ Department of Plastic and Reconstructive Surgery, Keio \\ University, Tokyo, Japan
}

We present the case of a patient with severe postoperative scarring from surgical treatment for gastroschisis, with the intestine located immediately under the dermal scar. Although many patients are unsatisfied with the results of scar repair treatment, few reports exist regarding severe or difficult cases involving the surgical repair of postoperative scar contracture. We achieved an excellent result via simulation involving graph paper drawings that were generated using computed tomography images as a reference, followed by dermal scar deepithelialization. The strategy described here may be useful for other cases of severe postoperative scar contracture after primary surgery for gastroschisis.

Keywords Gastroschisis / Scarring / Abdominal plasty / Congenital abdominal wall defect
Correspondence: Naohiro Ishii Department of Plastic and Reconstructive Surgery, Tochigi Cancer Center, 4-9-13, Yohnan, Utsunomiya City, Tochigi 320-0834, Japan

Tel: +81-28-658-5151

Fax: +81-28-658-5669

E-mail: ishinao0916@gmail.com

No potential conflict of interest relevant to this article was reported.

\section{INTRODUCTION}

Surgery to address gastroschisis is sometimes repeated several times, resulting in severe postoperative scar contractures. Many patients undergo scar repair treatment, but they are often unsatisfied with the results [1]. In contrast, previous case reports describe young patients with postoperative scarring after surgery for gastroschisis who underwent abdominoplasty and achieved good aesthetic results $[2,3]$. However, few reports have described multiple or severe cases involving the surgical repair of postoperative scar contractures. Here, we present the case of a patient with severe scarring following surgical treatment for gastroschisis and with a portion of the intestine located immediately under the dermal scar.

\section{CASE}

A 16-year-old girl underwent multiple operations for congenital gastroschisis at other hospitals. Primary skin closure was performed, and abdominal plasty was avoided. The patient presented with a complicated scar on her middle abdominal wall that measured approximately $5 \times 12 \mathrm{~cm}$, as well as other depressive scars resulting from drain insertions (Fig. 1A). Computed tomography (CT) images demonstrated that part of her intestine was located immediately under an extremely thin postoperative skin layer, with suspected adhesion. In addition, a partial defect of the rectus abdominis muscle was detected (Fig. 1B).

To perform a safe and effective operation, we positioned the skin incision to avoid intestinal trauma while considering the cosmetic elements related to postoperative scarring. In addition,

Copyright $($ C 2017 The Korean Society of Plastic and Reconstructive Surgeons

This is an Open Access article distributed under the terms of the Creative Commons Attribution Non-Commercial License (http://creativecommons.org/

licenses/by-nc/4.0/) which permits unrestricted non-commercial use, distribution, and reproduction in any medium, provided the original work is properly cited. I www.e-aps.org 
Fig. 1. Preoperative view, images, planning, and simulation

(A) The preoperative view of a 16-year-old girl's abdomen with severe scar contractures resulting from surgical treatment of congenital gastroschisis. (B) Preoperative computed tomography (CT) images. Part of the intestine is located immediately under, and is suspected to have adhered to, an extremely thin postoperative skin layer. In addition, the rectus abdominis muscle exhibited a partial defect. The blue arrow indicates the measured width of the skin located immediately above the intestine. The red arrow indicates the width of the weak fascia beyond which subcutaneous undermining should be performed. (C) Preoperative planning and simulation for surgical scar repair. Left: With reference to the CT image, we plotted the blue arrow on graph paper. Right: With reference to the CT image, we plotted the red arrow on graph paper and marked areas where the rectus abdominis muscle was present or absent (circle, present; triangle, inconclusive; $x$, absent). (D) Plotted graph papers were cut and traced on the patient's abdomen. The outline of the graph paper containing the blue line is marked in blue on her body; the black line on her body is outside of the blue line and indicates the incisional line on the skin. The outline of the graph paper containing the red line is marked in red on her body; this was effectively used as a reference during subcutaneous undermining.

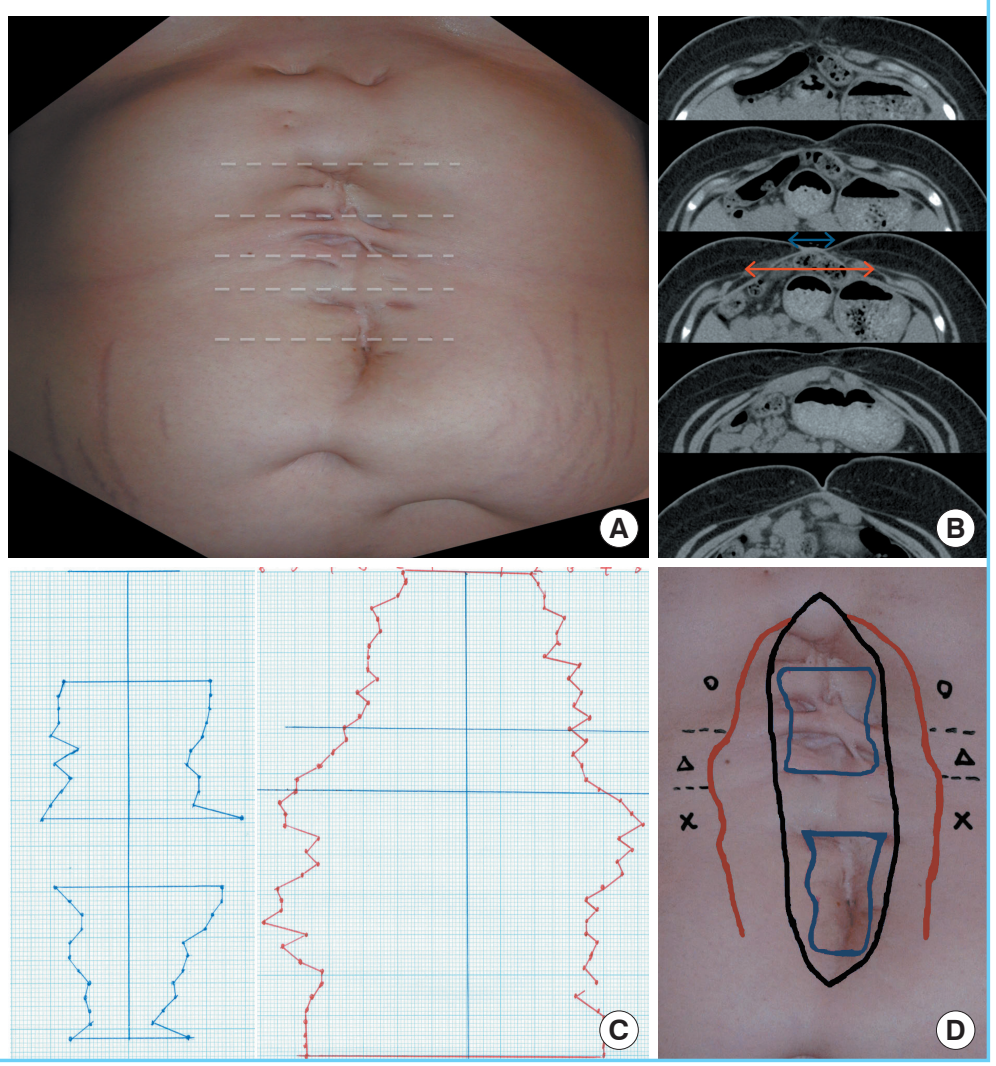

Fig. 2. Intraoperative photographs

(A) The blue ellipse indicates the area of de-epithelialized skin. (B) View of the surgical site immediately after the operation.
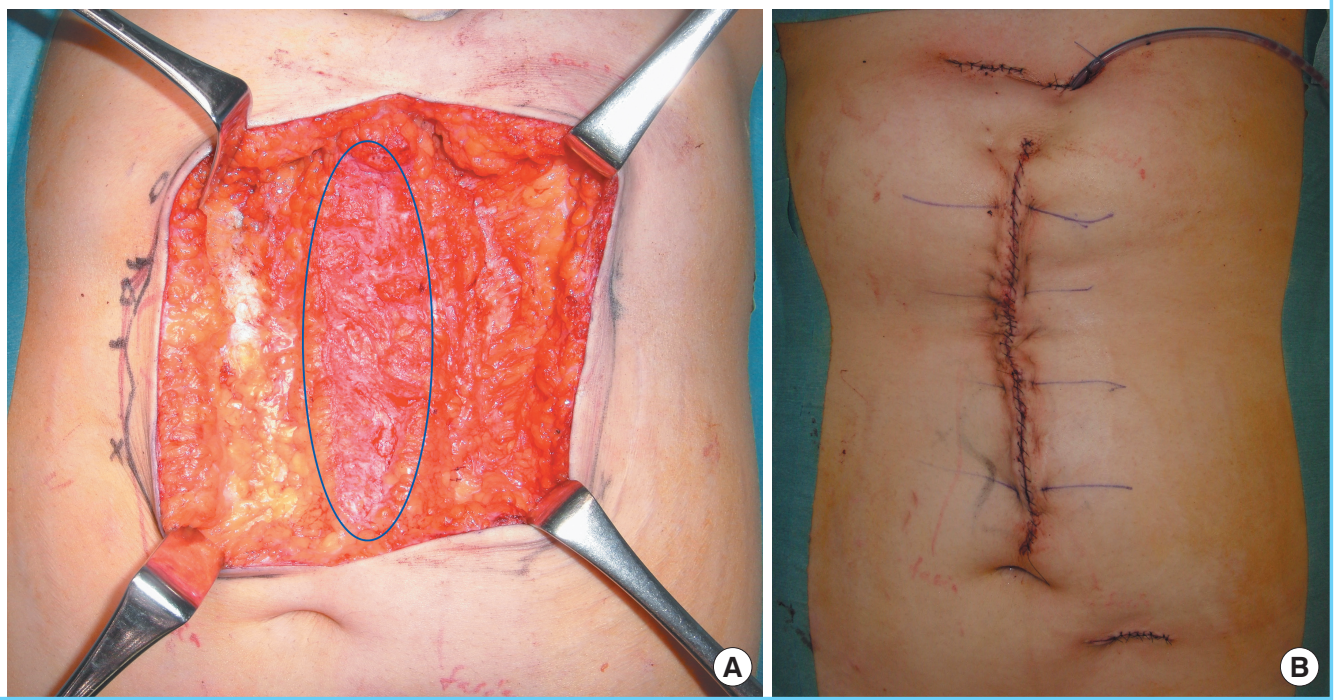

we conducted a detailed investigation of the range across which retention sutures could be placed while maintaining the structure of the fascia. Using 3-mm-slice CT images as references, the range of the intestine located immediately below the thin postoperative skin, the range of stable fascia structure and intended retention sutures, and the range of the rectus abdominis fascia muscle intended for preservation were measured and drawn on graph paper (Fig. 1C). The resulting shapes were cut from the paper, traced onto the patient's body, and used to determine the skin incisional line (Fig. 1D). The midline was designated from the xiphoid process to the umbilicus. We planned an operation involving the following elements: the range of intestine located 


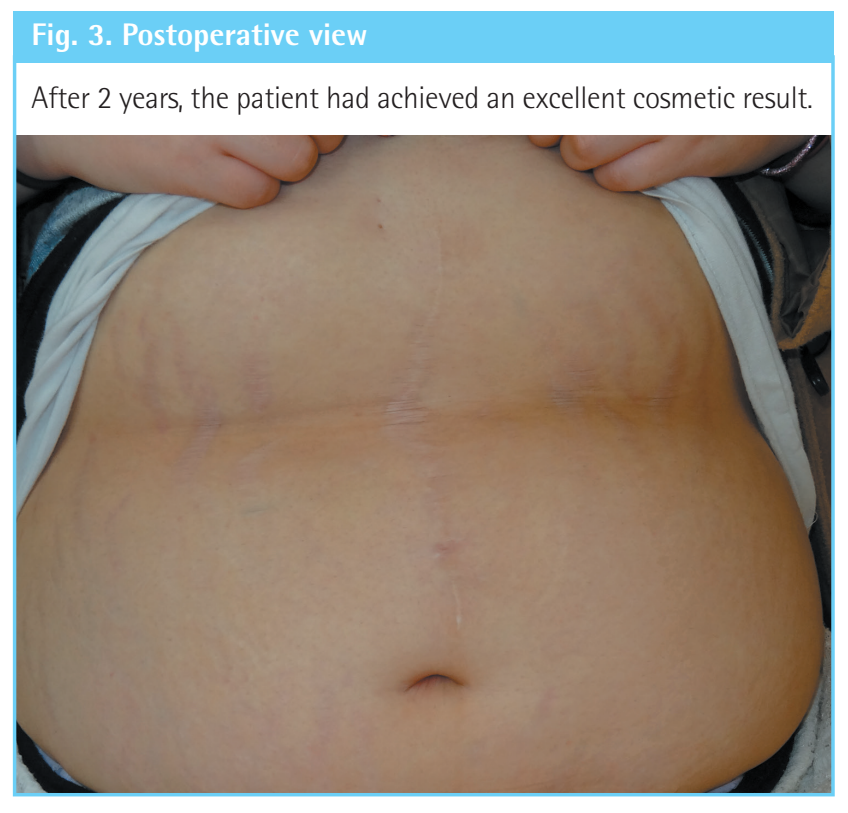

immediately below the thin postoperative skin layer; a skin incision that would allow subcutaneous tissue preservation; de-epithelialization of the skin only near the midline; and gradual, bilateral deep undermining of the subcutaneous tissue beyond the stable fascia structure with retention sutures as needed.

The above-described operation was performed with the patient under general anesthesia, as planned. The skin surrounding the incision was carefully de-epithelialized in the middle dermal layer, gradual deep undermining of the subcutaneous tissue was performed bilaterally, and the bilateral edge of the stable rectus abdominis fascia was approximated to the extent possible in order to reduce tension on the skin sutures at the midline. Subsequently, skin suturing was performed in a layerto-layer manner (Fig. 2). Three scars from previous drain inser- tion procedures were incised and sutured above the rectus abdominis fascia. One caudal scar was preserved as a substitute for the umbilicus. Intestinal trauma was avoided during surgery, and the patient did not experience postoperative complications. Two years later, she had achieved excellent cosmetic results (Fig. 3), with no exacerbation of herniation or ileus.

\section{DISCUSSION}

A detailed preoperative evaluation is important when performing scar revision surgery with the intent of avoiding laparotomy and subsequent trauma to the intestine located immediately under the dermal scar. In addition, a preoperative simulation performed using CT images, graph paper, and skin scar de-epithelialization can promote a good aesthetic result. The presented technique may also be applicable for use with tissue expanders when the part of the intestine located immediately under the dermal scar is extremely wide [3]. We hope that our strategy will be useful for surgeons planning treatment for cases of severe postoperative scar contracture after primary surgery for gastroschisis.

\section{REFERENCES}

1. Tunell WP, Puffinbarger NK, Tuggle DW, et al. Abdominal wall defects in infants. Survival and implications for adult life. Ann Surg 1995;221:525-8.

2. Pechter EA. Abdominoplasty in an adult survivor of gastroschisis. Ann Plast Surg 2006;56:327-9.

3. Fuentes S, Marti E, Delgado MD, et al. Management of the sequelae of severe congenital abdominal wall defects. Arch Plast Surg 2016;43:258-64. 